\title{
Time-Frequency Analysis and Singular Value Decomposition Applied to the Highly Multicomponent Musical Signals
}

\author{
Irena Orović, Srdjan Stanković and Andjela Draganić \\ Faculty of Electrical Engineering, University of Montenegro, Dzordza Vasingtona bb, 81000 \\ Podgorica, Montenegro \\ Corresponding author's email: andjelad@ac.me
}

\begin{abstract}
An algorithm for decomposition of highly multicomponent signals, with variable components energy, has been proposed. The algorithm combines the singular value decomposition with the suitable time-frequency analysis approach. The auto-correlation matrix is obtained by applying the inverse form of the cross-terms free time-frequency distribution. The decomposition of the time-frequency based auto-correlation matrix produces vectors that correspond to the individual signal components. The efficiency of the proposed algorithm has been tested on different signals.
\end{abstract}

KEYWORDS: time-frequency analysis, signal decomposition, musical signals

\section{INTRODUCTION}

The stationary spectrum is commonly analyzed by using the Fourier transform. However, in the case of non-stationary signals with variable spectral content, the Fourier transform does not provide information about the time instants when spectral components occur. It is the reason for employing the joint time-frequency analysis [1], [2]. Different timefrequency distributions have been used in real applications with non-stationary signals [3]-[7], such as radar, sonar, biomedical, communication signals, etc. The simplest time-frequency representation is obtained by using the short-time Fourier transform (STFT) and its energetic form spectrogram (SPEC). The STFT calculation is based on the Fourier transform of windowed signal. The resolution of time-frequency representation, obtained by using the STFT or spectrogram, depends on the window length and it is not possible to achieve good time and good frequency resolution simultaneously. In order to improve the resolution of 
spectrogram, quadratic time-frequency distributions have been introduced [3], [5]-[7], and later, more complex, higher order distributions [8], [9]. The commonly used quadratic distributions include the Wigner distribution, distributions from the Cohen class and the Smethod. When dealing with multicomponent signals, the S-method can provide efficient cross-terms free representation with good auto-terms concentration in the time-frequency domain [6], [10], [11]. Further, its realization can be simply performed by using the STFT. Also, the S-method has been successfully implemented in hardware, and thus, it has been used in many real-time applications.

One of the important time-frequency analysis applications assumes characterization of signal components and calculation of the total number of components for highly multicomponent signal. For that purpose, the time-frequency distribution can be combined with the principal component analysis (PCA) [12]-[14]. PCA has been introduced as a procedure based on the orthogonal transform, which produces a set of values of uncorrelated variables called principal components. PCA can be done by using the singular value decomposition. For example, the right and left singular vectors have been used with timefrequency analysis and neural networks for characterization and classification of EEG signals [14]. Another algorithm that is based on singular (eigen) decomposition is known as MUSIC [15]-[17]. MUSIC is frequency estimation method based on eigendecomposition of the covariance matrix [15]. It has been introduced in sensor array signal processing applications (radars), for direction of arrival (DOA) estimation [16], [17]. MUSIC splits $M$-dimensional space into the signal and the noise subspace. Frequencies are estimated from the pseudospectrum, formed by using eigenvectors. On the other side, we explore the idea that the eigenvectors of properly formed auto-correlation matrix correspond directly to the signal components [7]. For that purpose we need to use certain cross-terms free time-frequency representation to produce the corresponding cross-terms free autocorrelation matrix.

In this paper, the time-frequency analysis has been combined with singular value decomposition for the purpose of musical signal decomposition. The procedure starts from the cross-terms free time-frequency representation, which is used to obtain the suitable autocorrelation function as a result of inverse transform. The decomposition of the autocorrelation function produces separated signal components. The proposed approach treats the decomposition of highly multicomponent musical signals. However, note that we only aim to demonstrate the decomposition ability of the proposed algorithm, rather than to provide a 
complete music processing application. Moreover, we believe that this procedure may be the basis to build sophisticated music analysis/synthesis algorithms.

The paper is organized as follows. The theoretical background on the time-frequency signal analysis and quadratic distributions is given in Section II. The time-frequency based signal decomposition method is given in Section III. The algorithm for multicomponent signal decomposition is proposed in Section IV, while the experimental results are given in Section $\mathrm{V}$. The concluding remarks are given in Section VI.

\section{THEORETICAL BACKGROUND}

One of the oldest and commonly used time-frequency distribution is the Wigner distribution which has been defined as:

$$
W D(t, \omega)=\int_{-\infty}^{\infty} x\left(t+\frac{\tau}{2}\right) x^{*}\left(t-\frac{\tau}{2}\right) e^{-j \omega \tau} d \tau .
$$

For monocomponent signals, the Wigner distribution provides good concentration in the time-frequency plane, especially when the instantaneous frequency (IF) changes linearly. The resolution of the Wigner distribution is not so window dependent as it is the case with spectrogram. The discrete form of the Wigner distribution can be written as:

$$
W D(n, k)=\sum_{n=-N / 2}^{N / 2} x(n+m) x^{*}(n-m) e^{-j \frac{2 \pi}{N+1} 2 k m} .
$$

For multicomponent signals, Wigner distribution produces unwanted components called cross-terms. They appear between two signal terms (auto-terms) at the position of their arithmetic mean. In order to avoid the presence of cross-terms, the S-method has been introduced [6]. It provides the concentration of auto-terms as in the Wigner distribution, while reduces or eliminates the presence of cross-terms. The S-method is defined as follows [6]:

$$
\operatorname{SM}(t, \omega)=\frac{1}{\pi} \int_{-\infty}^{\infty} P(\theta) \operatorname{STFT}(t, \omega+\theta) \operatorname{STFT}^{*}(t, \omega-\theta) d \theta
$$

The convolution along the frequency axis improves the auto-terms concentration, but it should be performed only within the same auto-term, avoiding different signal components being convolved. It is realized using a frequency domain window, denoted by $P(\theta)$ $(P(\theta)=0$ for $|\theta|>L)$. Observe that for $P(\theta)=\pi \delta(\theta)$ and $P(\theta)=1$ the spectrogram and the Wigner distribution are obtained, respectively. 
The discrete form of the S-method, which is suitable for practical realizations, is defined as follows:

$$
\begin{aligned}
& \operatorname{SM}(n, k)=\sum_{l=-L}^{L} P(l) \operatorname{STFT}(n, k+l) \operatorname{STFT}^{*}(n, k-l)= \\
& =\operatorname{SPEC}(n, k)+2 \operatorname{Re}\left\{\sum_{i=1}^{L} \operatorname{STFT}(n, k+l) \operatorname{STFT}^{*}(n, k-l)\right\},
\end{aligned}
$$

where $n$ and $k$ denotes discrete time and frequency, respectively, while the rectangular

frequency domain window $P(l)$ is assumed. The cross-terms reduction will depend on the choice of parameter $L$. Namely, $L$ should be chosen such that $2 L+1$ is greater or equal to the auto-term width and less than the distance between the auto-terms.

Note that the cross-terms free S-method can be written as:

$$
S M(n, k)=\sum_{c=1}^{M} W D_{c}(n, k),
$$

where $M$ is the number of auto-terms, while $W D_{c}(n, k)$ is the Wigner distribution of the $c$-th auto-term.

\section{TIME-FREQUENCY ANALYSIS AND THE SINGULAR VALUE DECOMPOSITION}

\subsection{Principal Components Analysis based on the Singular Value Decomposition}

Singular value decomposition represents a method that transforms the original correlated variables into the uncorrelated set of variables. It allows us to identify the direction along which the data samples perform dominant variations. For a certain matrix $\mathbf{S}$, the singular value decomposition (SVD) is defined as follows:

$$
\mathbf{S}=\mathbf{U} \boldsymbol{\Sigma} \mathbf{V}^{\mathbf{T}},
$$

where $\boldsymbol{\Sigma}$ is a diagonal singular values matrix of the same size as $\mathbf{S}$, and the values are sorted in decreasing order along the main diagonal. The $\mathbf{U}$ and $\mathbf{V}$ are orthonormal matrices whose columns represent left and right singular vectors, respectively. If $\mathbf{S}$ is $M x N$ matrix $(M>N)$, then $\mathbf{U}$ is of size $M x M, \Sigma$ is $M x N$ matrix, while $\mathbf{V}$ is $N \mathrm{x} N$ matrix. This case is known as a full SVD. In order to provide more efficient method in terms of memory requirements, the economysized SVD can be computed as follows: 
- $\quad$ only $N$ columns of $\mathbf{U}$ is computed

- $\quad$ only $N$ rows of $\Sigma$ is computed.

The singular value decomposition has been used in numerous practical applications for characterization of signals and their components. The SVD has been applied on the timefrequency distributions to extract some specific features used for the signals characterization. Most of the procedures are based on the use of singular values [12], [13]. However, significant information about the patterns embedded in the matrix can be obtained using the left and right singular vectors, especially those corresponding to the largest singular values [14]. Namely, the left and right singular vectors contain the information about time and frequency domain information of the signal, respectively.

Here, the singular value decomposition is used to extract each individual signal component from the auto-correlation function. The auto-correlation function is obtained by applying the inversion of a suitable time-frequency distribution. As shown in the sequel, to ensure the absence of cross-terms when extracting the signal auto-components, the crossterms free S-method has been used.

\subsection{Component extraction by using the SVD and the S-method}

The inverse Wigner distribution for a separated $c$-th signal component can be obtained from (2) having that $x(n)=x_{c}(n)$ :

$$
x_{c}(n+m) x_{c}^{*}(n-m)=\frac{1}{N+1} \sum_{k=-N / 2}^{N / 2} W D_{c}(n, k) e^{j \frac{2 \pi}{N+1} k 2 m} .
$$

By replacing $n+m=p$ and $n-m=q$, we get [7]:

$$
x_{c}(p) x_{c}^{*}(q)=\frac{1}{N+1} \sum_{k=-N / 2}^{N / 2} W D_{c}\left(\frac{p+q}{2}, k\right) e^{j \frac{2 \pi}{N+1}(p-q) k} .
$$

The left hand side corresponds to the auto-correlation matrix:

$$
R_{c}(p, q)=x_{c}(p) x_{c}^{*}(q)
$$

where $x(p)$ is column vector, whose elements are signal terms, and $x *(q)$ is row vector, with complex conjugate elements. For a sum of $M$ signal components (8) becomes: 


$$
\sum_{c=1}^{M} R_{c}(p, q)=\frac{1}{N+1} \sum_{k=-N / 2}^{N / 2} \sum_{c=1}^{M} W D_{c}\left(\frac{p+q}{2}, k\right) e^{j \frac{2 \pi}{N+1}(p-q) k}=\frac{1}{N+1} \sum_{k=-N / 2}^{N / 2} S M\left(\frac{p+q}{2}, k\right) e^{j \frac{2 \pi}{N+1}(p-q) k} .
$$

The singular value decomposition is applied to the auto-correlation matrix as follows:

$$
R(p, q)=\sum_{c=1}^{M} R_{c}(p, q)=U \Sigma V^{T} .
$$

Furthermore, in order to separate the exact signal components, let us observe the case when the time-frequency distribution is represented by a square matrix, i.e. the time and frequency dimensions are the same. The auto-correlation function $R(p, q)$ is the symmetric square matrix with respect to the main diagonal, and thus we may consider $U=V$ as the eigenvectors and $\Sigma=\Lambda$ as the eigenvalue matrix.

Hence, the decomposition problem is reduced to the calculation of eigenvectors, such that each eigenvector corresponds to one separated signal component. The auto-correlation matrix $R(p, q)$ can be decomposed as follows:

$$
R(p, q)=\sum_{c=1}^{M} R_{c}(p, q)=\sum_{j=1}^{M} \lambda_{j} u_{j}(n) u_{j}^{*}(n),
$$

where $\lambda_{j}$ are eigenvalues and $u_{j}(n)$ are eigenvectors of the autocorrelation matrix $R$. Note that the obtained eigenvectors corresponds to the selected $K$ signal components, while the eigenvalues are related to the components energy.

\section{DECOMPOSITION OF MUSICAL SIGNALS}

Different examples of highly multicomponent signals can be found among musical tones produced by various acoustic instruments. The analysis of frequency and amplitude envelope of musical signal has been the topic in various papers [18]. The spectral characteristics of these signals depend on the tones, but also can vary depending on the sound level, playing dynamic level, etc. For instance, the louder tones have brighter timbre [19]. The spectral characteristics of different tones played on the same level can be quite different. Also, even the same tones played at different exemplars of the same instrument may have certain specificity in the spectrum. Extracting individual components allows accurate analysis, but also various modifications of musical tones [18], [19]. The Fast Harmonic Matching Pursuit algorithm has been employed in [20] to approximate audio signals as a combination of $M$ 
harmonic structures. The method provides efficient results in decomposition of musical recordings, but it is sensitive to its parameters values. In some cases, the separation of harmonic components can be done by filtering in the spectral domain. The frequencies of each harmonic have to be known, and thus it is necessary to estimate signal frequencies by applying, for example, tracking harmonics, peak-picking, etc [21], [22].

In this Section we proposed another solution for decomposition of highly multicomponent musical signals. Unlike the previously described algorithms that explore the harmonic properties of signals, the proposed procedure does not use any a priori knowledge on the signal nature. Hence, it can be efficiently used for both harmonic and non-harmonic data. The algorithm is based on the previously presented eigendecomposition, but the straightforward use is not applicable, having in mind that the energy of components may vary significantly. Thus, we propose a modification which could be applied to different types of musical (or other similar) signals. The algorithm for the proposed decomposition is shown in Figure 1. The decomposition is done in several iterations. In each iteration, we treat the components within the regions with approximately the same energy.

Let $x(t)$ be the signal on which the decomposition should be applied, while its Fourier transform is denoted by $X(\omega)$. The parameter $K$ is set according to the number of components that we aim to separate within the observed iteration.

In the first iteration the S-method is calculated for the whole signal and decomposition is applied as:

$$
R=\frac{1}{N+1} \sum_{k=-N / 2}^{N / 2} S M\left(\frac{p+q}{2}, k\right) e^{j \frac{2 \pi}{N+1}(p-q) k}=\sum_{j=1}^{K} \lambda_{j} u_{j}(n) u_{j}^{*}(n) .
$$

Note that the number of separated components is actually $K / 2$, since the components appear in pairs due to the spectrum symmetry. In the next step, the S-method is calculated by using the eigenvectors $u_{j}$ for each separated $j$-th component:

$$
S M_{j}(n, k)=\sum_{l=-L_{j}}^{L_{j}} \operatorname{STFT}_{j}(n, k+l) \operatorname{STFT}_{j}^{*}(n, k-l),
$$

where $j=1,2, \ldots, K / 2$. Then the central frequency of the $j$-th component in the first iteration is determined as:

$$
\omega_{j}=\underset{k}{\operatorname{argmax}}\left\{S M_{j}(n, k)\right\}
$$

After identifying the components positions, the corresponding frequency region is set to zero within the Fourier transform $X(\omega)$ of the signal: 


$$
X\{\omega\}=\left\{\begin{array}{l}
0, \text { if } \omega \in\left\{\left(\frac{\omega_{c}}{2}-\frac{m}{2 a} \omega_{j}\right) \pm \Delta \omega, \frac{m}{a} \omega_{j}+\left(\frac{\omega_{c}}{2}-\frac{m}{2 a} \omega_{j}\right) \pm \Delta \omega\right\}, \\
X\{\omega\}, \text { otherwise }
\end{array}\right.
$$

where $m$ and $a$ are window lengths used to calculate the STFT within the $S M$ and $S M_{j}$, respectively. The inverse Fourier transform is used to obtain the signal for the next iteration:

$$
x_{i+1}(t)=i f f t(X(\omega)) .
$$

The algorithm is then repeated in the next iteration by using the signal $x_{i+1}(t)$. Theoretically, the procedure ends once we remove the whole frequency content from $X(\omega)$, i.e., when $X(\omega)=0$. However, in practice, the noise is usually present within the real signals. Hence, instead of $X(\omega)=0$, the condition is defined as $X(\omega)<\xi$. The floor value $\xi$ is determined empirically and it is approximately equal to the peak noise energy within $X(\omega)$.

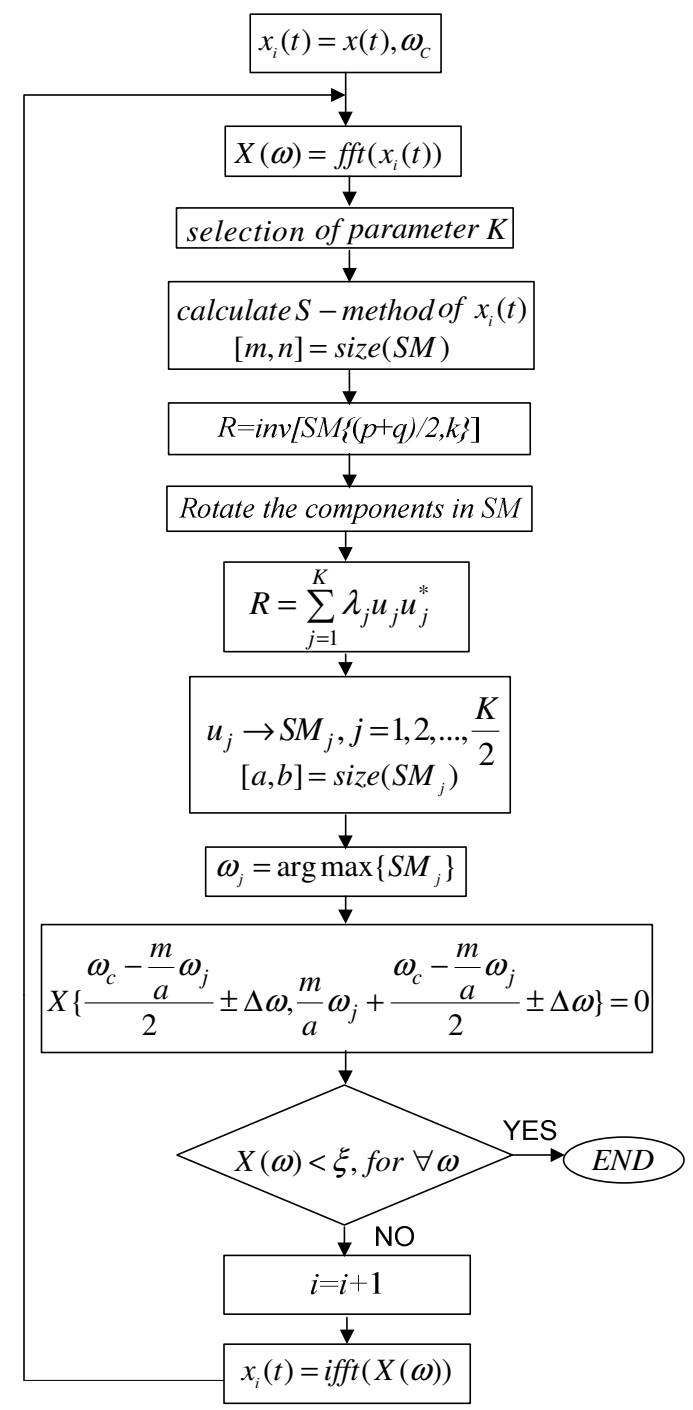

Figure 1: The proposed algorithm for time-frequency based decomposition 


\section{EXPERIMENTAL RESULTS}

\subsection{Example: Flute and violin signals}

The proposed procedure is firstly applied on a flute signal. The signal segments with 1000 samples are used in the analysis. The parameter $L$ for the S-method calculation is set to the value 6 , in order to provide good concentration of components and, at the same time, to avoid the cross-terms. The S-method of flute signal is shown in Figure 2. The signal consists of nine components. Note that the energy of components varies significantly, depending on the frequency at which component appears, as it can be seen from the Figure 2 (the S-method is shown in the log scale, because the components at higher frequencies (upper part) have several times lower energy than the low-frequency components).

The highest energy components are separated in the first iteration. In our experiments, it has been shown that an optimal number of components that should be extracted in each iteration is $K=4$. Although for most of the tested signals even larger $K$ can be used, we prefer to choose an optimal value of $K$ that should be used for any signal type (even for some very specific cases). Hence, in all iterations $K$ equal to 4 is considered. The components extracted through the five iterations are illustrated in Figure 3.

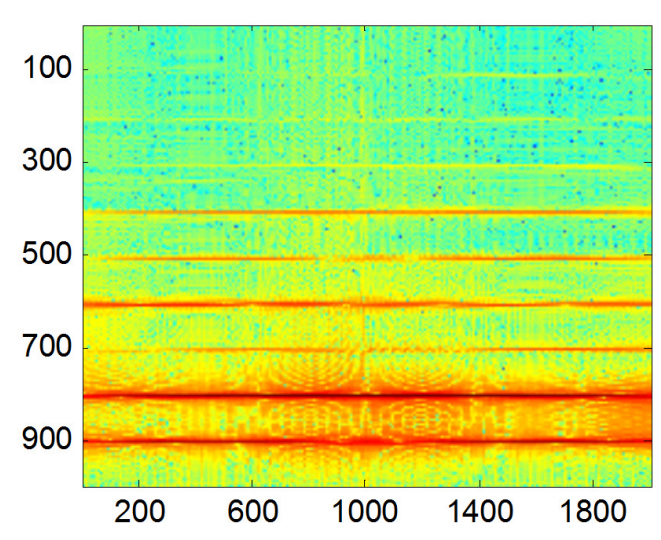

Figure 2: The S-method of a sample flute signal 

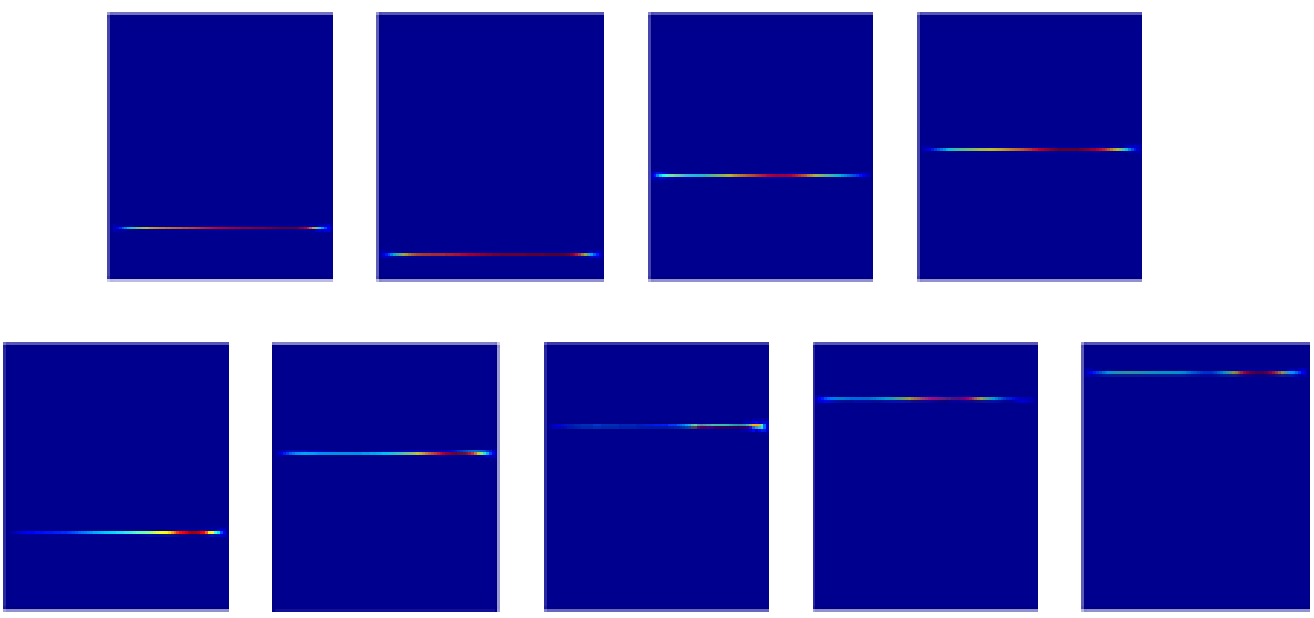

Figure 3: Flute signal components (on positive frequencies) separated in 5 iterations

The proposed algorithm has been successfully applied even to a more complex signal structures. For instance, let us observe the violin signal that consists of a large number of closely spaced components as illustrated in Figure 4. Note that the distance between the individual components in the time-frequency plane is smaller than in the case of flute signal. The decomposition procedure is applied in the same way and with the same values of parameters as in the previous example. As shown below, all 19 components are successfully extracted within 10 iterations and separated components are illustrated in Figure 5.

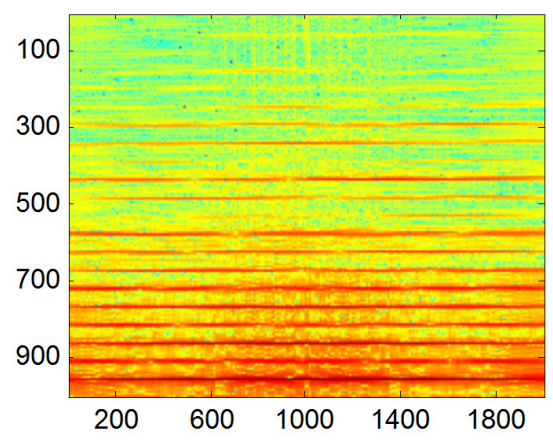

Figure 4: The S-method of a sample violin signal 

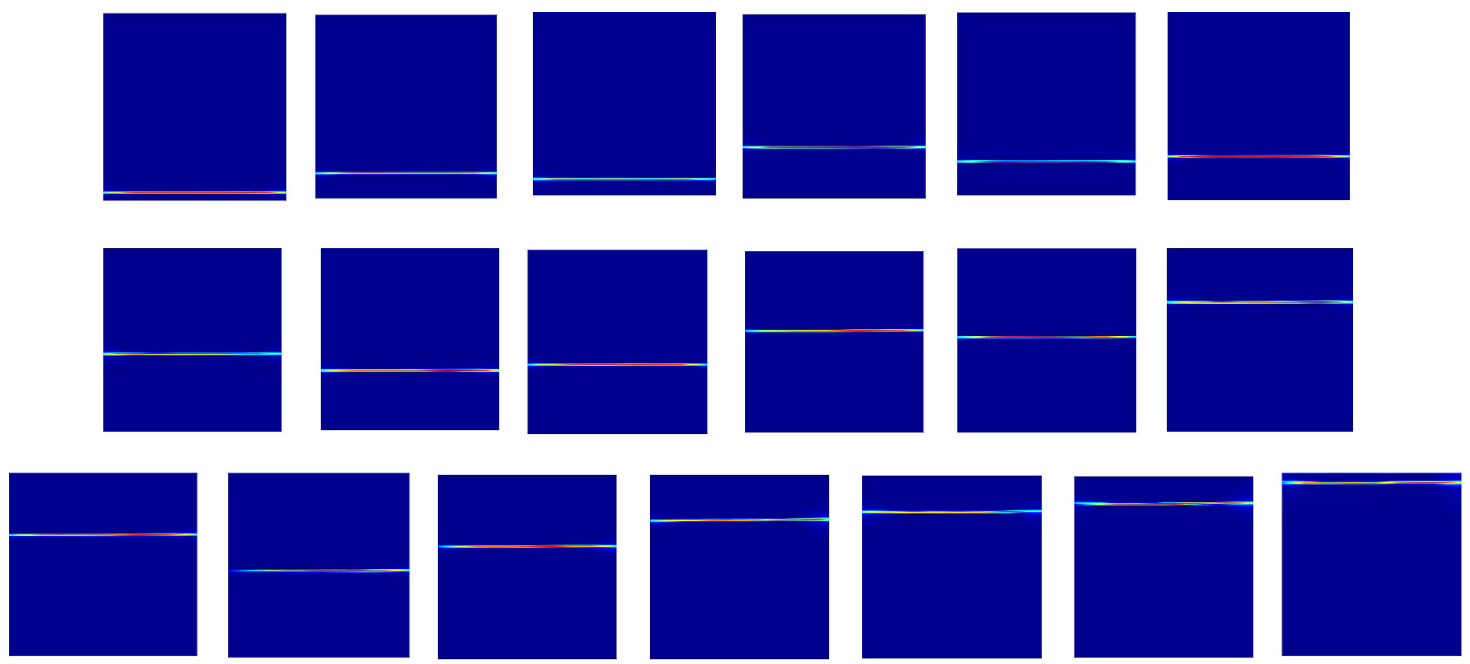

Figure 5: Separated violin signal components (at positive frequencies) within 10 iterations

Comparison: For the purpose of the comparison, in the sequel we consider the application of MUSIC algorithm for signal components separation. The flute signal from Figure 2 is observed. MUSIC pseudospectrum and discrete Fourier transform (DFT) of the considered signal are shown in Figure 6. We might note that the peaks in the MUSIC pseudospectrum do not correspond properly to the signal's DFT components. In order to apply the MUSIC algorithm, we need to specify the number $P$ of sinusoidal components within the signal.

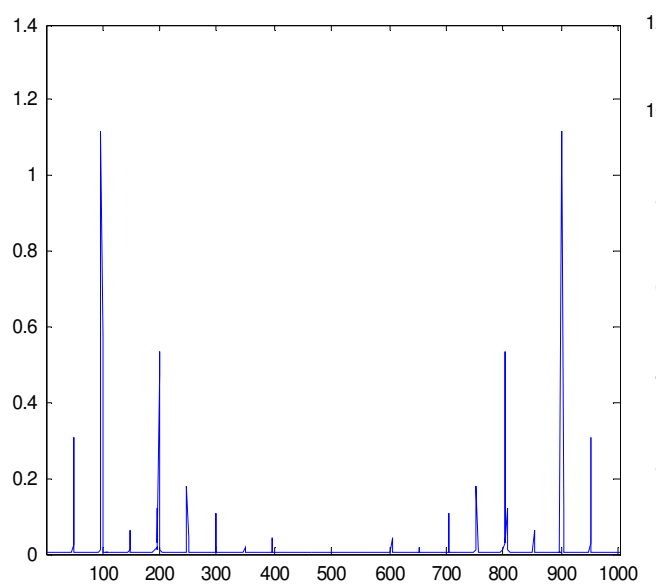

a)

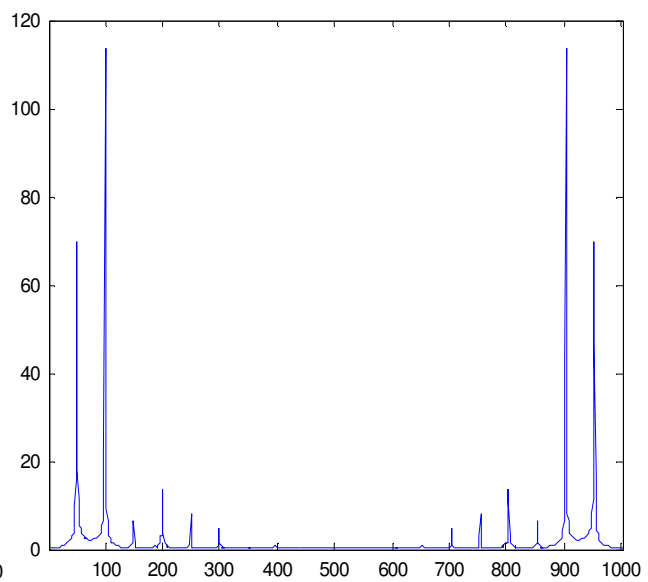

b)

Figure 6: a) MUSIC pseudospectrum, b) Discrete Fourier transform of the flute signal

Even if we know the number of components (e.g., $P=9$ for the observed flute signal), which is usually not the case in the practice, we are not able to extract all components. Namely, only the first 6 eigenvectors, obtained using MUSIC, correspond to the signal 
components (Figure 7a). The remaining eigenvectors are either parts of the same 6 components or belong to the noise (Figure 7b). As signal consists of 9 components, 3 of them cannot be detected using MUSIC. The MUSIC performance does not improve even if we increase the value of $P$. Additional drawback of the MUSIC algorithm, when applied to components separation, is the resolution of components which is dependent on the timewindow width.
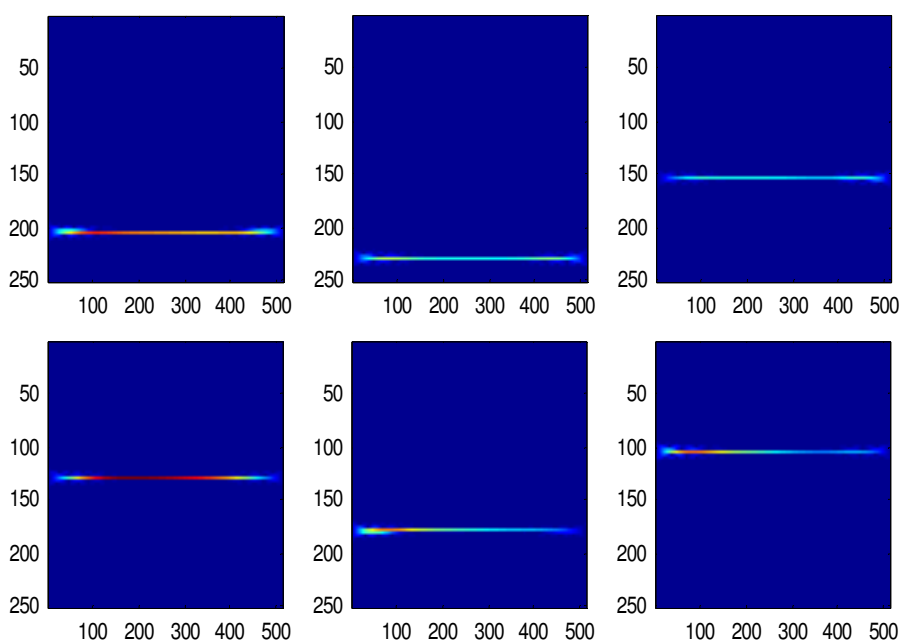

a)
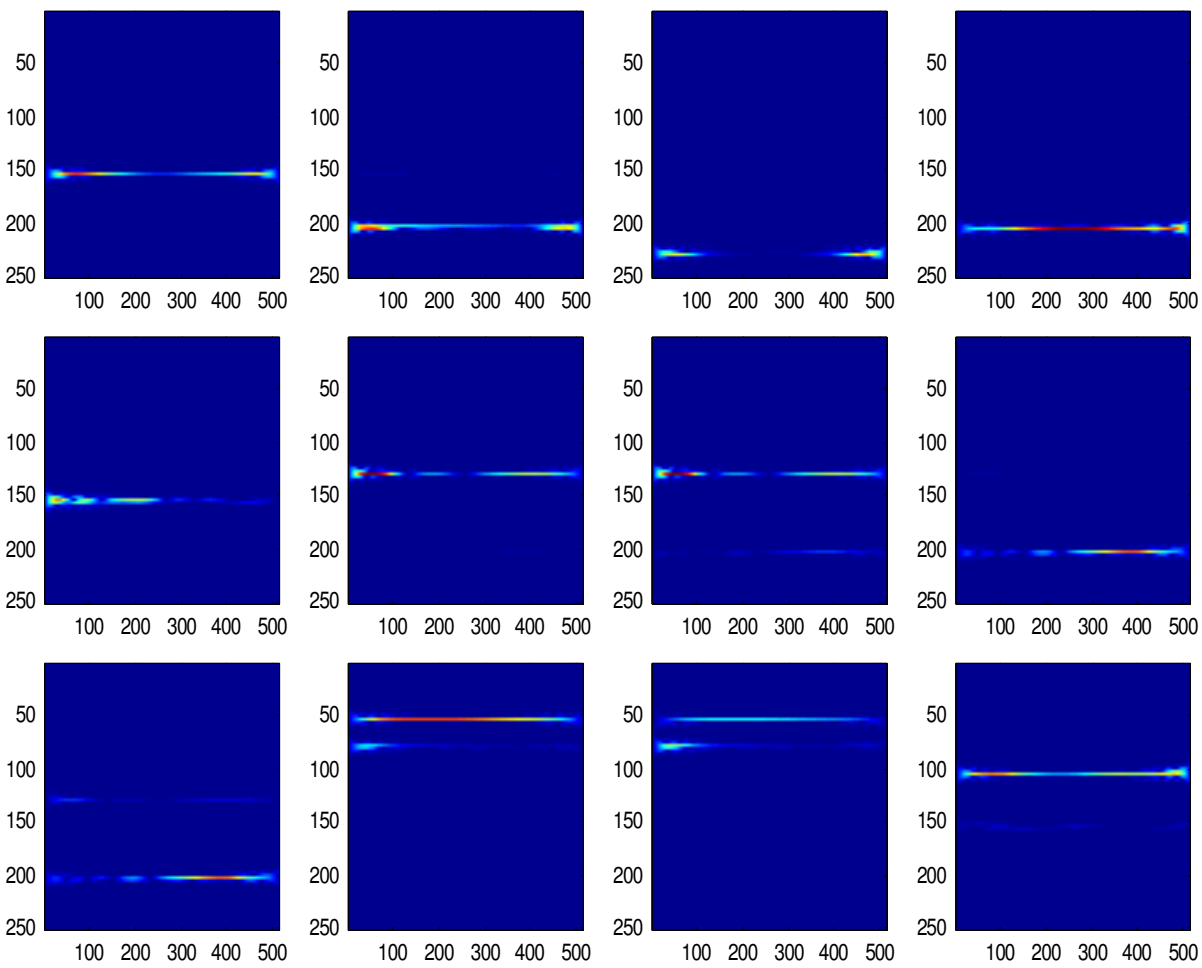

b)

Figure 7: Separated components by using MUSIC algorithm 


\subsection{Example: Performance of the proposed algorithm in the presence of noise}

The performance of the proposed algorithm is tested in the presence of noise. Namely, several experiments with different realizations of signal to noise (SNR) ratio have been performed. It has been shown that the decomposition method provide satisfactory results as long as the noise is not dominant over the components energy. Namely, high frequency components, due to their low energy, could be lost within the strong noise. Our experiments have shown that the decomposition (even on the high frequencies) is successful as long as the SNR is above $15 \mathrm{~dB}$. However, by increasing the noise strength, the weakest high-frequency components cannot be separated from the noise. The examples of noisy flute signal (for different SNRs) are shown in Figure 8, while the number of successfully separated components in terms of SNR is given in Table 1.

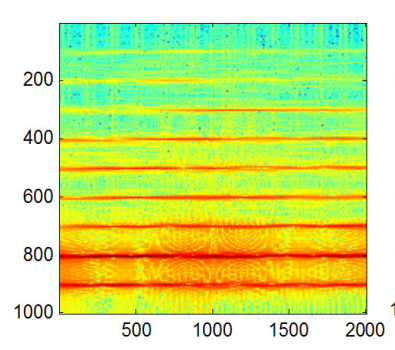

a)

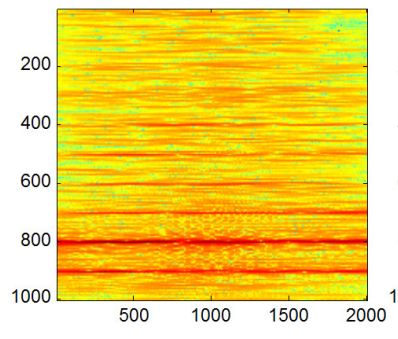

c)



b)

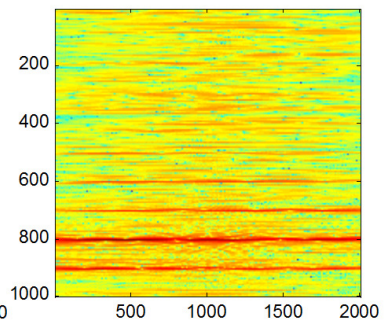

d)

Figure 8: a) Non-noisy signal, b) Noisy signal $S N R=15 d B$, c) Noisy signal $S N R=12 d B, d$ )

Noisy signal $\mathrm{SNR}=9 \mathrm{~dB}$ 
Table 1. The number of extracted components depending on the noise level

\begin{tabular}{|c|c|}
\hline Noise level & Number of extracted components (out of 9) \\
\hline $\mathrm{SNR}=9 \mathrm{~dB}$ & 7 \\
\hline $\mathrm{SNR}=12 \mathrm{~dB}$ & 8 \\
\hline $\mathrm{SNR}=14 \mathrm{~dB}$ & 8 \\
\hline $\mathrm{SNR}=15 \mathrm{~dB}$ & 8 \\
\hline $\mathrm{SNR}>15 \mathrm{~dB}$ & 9 \\
\hline
\end{tabular}

\subsection{Example: Performance of the proposed algorithm for non-harmonic signal}

In the sequel, the results of decomposition procedure applied to the non-harmonic signal are shown. The signal consists of 12 components, with different durations, different energies and different frequency gaps (distance between different components). Time-frequency representation of the non-harmonic signal is shown in Figure 9. The parameter $L$ within the Smethod calculation is set to the value 6 , whereas 2 components are extracted in each iteration $(K=4)$. It can be seen that the procedure provides the same performance as in the case of other considered signals, which proofs that the proposed approach does not require harmonic structure. Separated components are shown in Table 2.

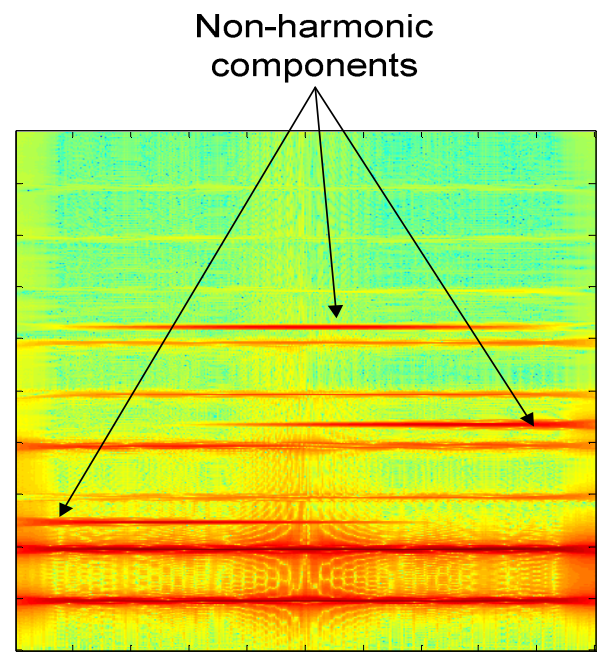

Figure 9: Time-frequency representation of the non-harmonic signal 
Table 2. Separated components of the non-harmonic signal within sixth iterations

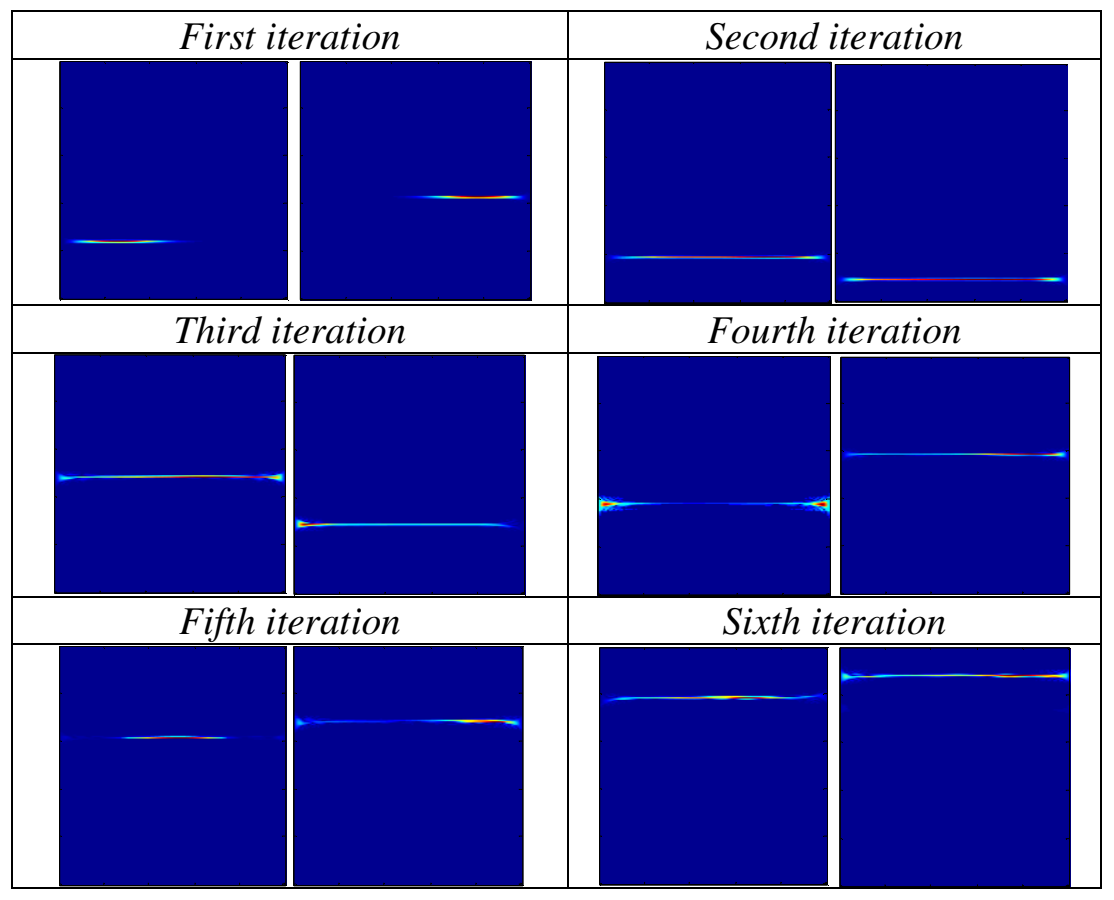

\section{CONCLUSION}

This paper presents an efficient iterative procedure for decomposition of signals with closely spaced components characterized by different energy levels. The algorithm is based on the time-frequency analysis combined with the singular value decomposition method. As a suitable time-frequency distribution, the S-method has been used to provide a cross-terms free representation. Due to the signal complexity the decomposition procedure is modified and applied within several iteration steps. In this way, even the components with lowest energy, that are almost invisible within the time-frequency representation, are detected and extracted. The efficiency of the procedure is shown in the examples with highly multicomponent flute and violin signals, as well as signals having non-harmonic structures.

\section{ACKNOWLEDGEMENT}

This work is supported by the Ministry of Education and Science of Montenegro. 


\section{REFERENCES}

[1] L. Cohen, Time-Frequency Distributions - A Review, Proceedings of the IEEE, 77(7), pp. 941-981., 1989.

[2] B. Boashash, Time-Frequency Signal Analysis and Processing: A Comprehensive Reference, ed., Amsterdam: Elsevier, 2003.

[3] F. Hlawatsch, G. F. Boudreaux-Bartels, Linear and Quadratic Time-Frequency Signal Representation, IEEE Signal Processing Magazine, 9 (2), pp. 21-67, 1992.

[4] E Sejdić., I Djurović., and J. Jiang: Time-frequency feature representation using energy concentration: An overview of recent advances, Digital Signal Processing, 19 (1), pp. 153 $183,2009$.

[5] P. Honeine, C. Richard, P. Flandrin, and J-B. Pothin: Optimal selection of time-frequency representations for signal classification: A kernel target alignment approach, ICASSP, 3(2006), pp. 476-479, May 2006.

[6] LJ. Stankovic, A method for time-frequency signal analysis, IEEE Transactions on Signal Processing, 42 (1), pp. 225-229., 1994.

[7] LJ. Stankovic, T. Thayaparan, M. Dakovic, Signal Decomposition by Using the S-Method with Application to the Analysis of HF Radar Signals in Sea-Clutter, IEEE Transactions on Signal Processing, 54 (11), pp. 4332- 4342, 2006.

[8] S. Stankovic, I. Orovic, C. Ioana, Effects of Cauchy Integral Formula Discretization on the Precision of IF Estimation: Unified Approach to Complex-lag Distribution and its LForm, IEEE Signal Processing Letters, 16 (4), pp. 307-310, 2009.

[9] S. Stankovic, N. Zaric, I. Orovic, C. Ioana, General form of time-frequency distribution with complex-lag argument, Electronics Letters, 44 (11), pp. 699-701, 2008.

[10] I. Orovic, S. Stankovic, Time-Frequency-Based Speech Regions Characterization and Eigenvalue Decomposition Applied to Speech Watermarking, EURASIP Journal on Advances in Signal Processing, Special Issue on Time-Frequency Analysis and its Application to Multimedia signals, Vol. 2010, Article ID 572748, 10 pages. 
[11] S. Stankovic, About Time-Variant Filtering of Speech Signals with Time-Frequency Distributions for Hands-Free Telephone Systems, Signal Processing, 80 (9), pp. 1777-1785, 2000.

[12] N. M. Marinovic and G. Eichmann, Feature Extraction and Pattern Classification in space/spatial-frequency domain, SPIE Intelligent Robots and Computer Vision, Proceedings of SPIE, 579, pp.1-25, 1985.

[13] S. Gu, J. Ni and J. Yuan, Non-stationary Signal Analysis and Transient Machining Process Condition Monitoring, International Journal of Machine Tools and Manufacture, 42 (1), pp. 41-51, 2002.

[14] H. Hassanpour, M. Mesbah and B. Boashash, SVD-Based Time-Frequency Feature Extraction for Newborn EEG Seizure, EURASIP Journal on Advances in Signal Processing, 2004:16, pp. 2544-2554.

[15] D. Manolakis, V. K. Ingle and S. M. Kogon, Statistical and Adaptive Signal Processing: Spectral Estimation, Signal Modeling, Adaptive Filtering and Array Processing, Artech House Signal Processing Library, April 30, 2005 I ISBN-10: 1580536107 I ISBN-13: 978-1580536103.

[16] R. O. Schmidt, Multiple emitter location and signal parameter estimation, Antennas and Propagation, IEEE Transactions on, Vol. 34, No. 3, pp. 276, 280, Mar 1986.

[17] Raed M. Shubair, Mahmoud A. Al-Qutayri and Jassim M. Samhan, A Setup for the Evaluation of MUSIC and LMS Algorithms for a Smart Antenna System, Journal of Communications, Vol. 2, No. 4 (2007), 71-77, Jun 2007.

[18] V. Valimaki, M. Ilmoniemi and M. Huotilainen, Decomposition and modification of musical instrument sounds using a fractional delay all pass filter, Proceedings of the 6th Nordic Signal Processing Symposium, 2004. NORSIG 2004, pp. 208 - 211.

[19] M. Fabiani, A method for the modification of acoustic instrument tone dynamics, Proceedings of the 12th International Conference on Digital Audio Effects (DAFx09). Como, Italy, 2009.

[20] R. Gribonval, E. Bacry, Harmonic Decomposition of Audio Signals with Matching Pursuit, IEEE Transactions on Signal Processing, 51 (1), pp. 101-111, 2003. 
[21] M.R. Every, Separating harmonic and inharmonic note content from real mono recordings, Proc. Digital Music Research Network Summer Conf., (Glasgow, U.K.), pp. 9-13, July 2005.

[22] M. R. Every, J. E. Szymanski, Separation of synchronous pitched notes by spectral filtering of harmonics, Audio, Speech, and Language Processing, IEEE Transactions on, Vol.14, No.5, pp.1845,1856, Sept. 2006. 